\title{
Effect of Background Magnetic Field on Type-II Superconductor under Oscillating Magnetic Field Simulated Using Ginzburg-Landau Model
}

\author{
Hasnain Mehdi Jafri, ${ }^{1}$ Congpeng Zhao, ${ }^{1,2}$ Houbing Huang $\mathbb{D}^{1,},{ }^{1,3}$ and Xingqiao Ma ${ }^{1}{ }^{1}$ \\ ${ }^{1}$ Department of Physics, University of Science and Technology Beijing, Beijing 100083, China \\ ${ }^{2}$ ASIC, China Center for Information Industry Development, Beijing 100083, China \\ ${ }^{3}$ Advanced Research Institute of Multidisciplinary Science, Beijing Institute of Technology, Beijing 100081, China \\ Correspondence should be addressed to Xingqiao Ma; xqma@sas.ustb.edu.cn
}

Received 8 August 2018; Accepted 27 September 2018; Published 12 November 2018

Academic Editor: Joseph S. Poon

Copyright ( 2018 Hasnain Mehdi Jafri et al. This is an open access article distributed under the Creative Commons Attribution License, which permits unrestricted use, distribution, and reproduction in any medium, provided the original work is properly cited.

\begin{abstract}
Cubic superconducting sample was simulated using time-dependent Ginzburg-Landau model under oscillating magnetic field with and without additional background static magnetic field. Vortex dynamics including entrance and exit from the sample was simulated. Magnetization and carrier concentration densities of the sample were studied as a function of external magnetic field variations. Anomalies in carrier concentration density were observed at certain values of the magnetic field which were correlated with the entrance and exit processes of vortices. Area swept by superconductor magnetization with magnetic field was observed to have a hysteresis-like behavior where area representing energy dissipated per cycle. This energy accumulation was suggested to cause instability in superconductor over the number of cycles and may result in thermal quenching. Temporal distribution of energy components showed consistency with the pattern observed for carrier concentration and magnetization under oscillating magnetic field. Rapid phase changes with magnetic oscillations resulted in oscillations in energy components, and irregular peaks and ripples in superconducting energy represent the situation of exit and entry of vortices. While the rise in interaction energy with cycles is referred to vortex relaxation time in a cycle, this energy is expected to accumulate and take other forms (e.g., heat) and is predicted to cause thermal quenching. In the presence of background static magnetic field, this energy dissipation was calculated to increase significantly while superconductor is subjected to oscillating magnetic field.
\end{abstract}

\section{Introduction}

In general, the behavior of superconductor in applied electric and magnetic field is attributed to the vortex dynamics in it, which generally results in energy dissipation (e.g., heat) and resulting thermal quenching of superconductivity. According to Bardeen-Cooper-Schrieffer (BCS) theory, spin singlet state (opposite spin electron pair), bound by phonon interaction, constitutes the ground state of condensate [1]. The proposed macroscopic quantum theory by Ginzburg and Landau (named Ginzburg-Landau (GL) theory) used Higgs mechanism of spontaneous symmetry breaking and quartic potential [2-5] to generate a local mass term of vector potential. Development of homogeneous ferromagnetic spins destroys the superconductivity if it exceeds critical magnetic field of the superconductor. This model successfully describes the Meissner-Ochsenfeld effect [4, 6-8]. The prediction of penetrating strong magnetic fields in type-II superconductors by Abrikosov [9] gave further credibility to GL model which was later verified experimentally [10-12]. Over the past 70 years, not only macroscopic properties of superconductors (such as categorizing superconductors in type-I and type-II, and description of vortex state in type-II superconductors [9]) but also mesoscopic superconducting samples were successfully described using this theory. GL model is probably the most accurate phenomenological model to describe macroscopic properties of superconductors [13, 14]. Gorkov [15] proved that close to critical temperatures microscopic 
BCS theory also reduces to GL theory. Various numerical methods, including finite difference [16-18], finite element [19-21], and spectral method [22], have been developed for the solution of this model. In this report, we used the semiimplicit finite difference method with staggered grid scheme.

Generally, high-temperature superconductor (HTS) based magnetic Levitation (maglev) vehicle systems consist of HTS on a permanent magnet (PM) guideway [23-25]. In general, uniform magnetic field is considered for PM guideways but practically this is not the case; there are situations of magnetic field variations (e.g., cracks, magnetic contacts, and structural and magnetic defects) resulting in a variable magnetic field. The frequency of this variation depends on the speed of the HTS on PM guideway (i.e., speed of the vehicle). The magnetic field in such a case is not exactly oscillating (which was reported earlier [18]), but it is (practically) a constant magnetic field having small jitters (or oscillations) in its amplitude appearing due to imperfections in PM guideway. In this work, we studied such a magnetic field and compared the results with results reported earlier for perfectly oscillating magnetic fields [18]. To investigate this type of energy loss, we studied the behavior of type-II superconductor exposed to an oscillating magnetic field simulated using time-dependent Ginzburg-Landau (TDGL) equations $[15,26,27]$ near critical temperature with and without an additional static background magnetic field. The dynamics of Abrikosov vortices in oscillating magnetic field is studied. A hysteresis-like behavior of sample magnetization was observed under oscillating magnetic fields, while background static magnetic field plays a vital role in thermal quenching of superconductor. The behavior of different energy components is also discussed in detail.

\section{Ginzburg-Landau Theory}

Primary variables in GL model are order parameter, $\psi$, and magnetic vector potential, $A$, occupying a superconductor sample in a three-dimensional region $\Omega$ having boundary $\Gamma$. In nondimensional state, these primary variables are linked to physical quantities, given as follows:

\section{Density of superconducting charge carriers, $n_{s},|\psi|^{2}$ Induced magnetic field, $\boldsymbol{\nabla} \times \boldsymbol{A}$ \\ Current density, $J=\nabla \times \nabla \times A$.}

Ginzburg-Landau free energy is taken from Gorkov and Eliashberg [28] given as follows:

$$
\begin{aligned}
G(\psi, \boldsymbol{A})= & \frac{1}{2 m}|(\hbar \nabla-\imath e \boldsymbol{A}) \psi|^{2}+\alpha|\psi|^{2} \mp \frac{1}{2} \beta|\psi|^{4} \\
& +\frac{1}{2 \mu_{o}}\left|\boldsymbol{\nabla} \times \mathbf{A}-\mu_{o} \boldsymbol{B}\right|^{2}
\end{aligned}
$$

where $e$ is charge and $m$ is effective mass of superconducting charge carriers, $\boldsymbol{B}$ is external magnetic field, $\hbar$ and $\imath$ are reduced Plank's constant (i.e., h/2 $\pi$ ), and $\sqrt{-1}$, while $\alpha$ and $\beta$ are phenomenological parameters depending on environmental factors (e.g., temperature).
We introduce London penetration depth $(\lambda=$ $\left.\sqrt{m c^{2} \beta / 16 \pi e^{2}|\alpha|}\right)$ and coherence length $(\xi=h / 2 \pi \sqrt{2 m|\alpha|})$ as length scale parameters. We introduce a length scale $l$ and nondimensionalized physical quantities, special and time coordinates $x(x=x, y, z)$ and $t$, order parameter $\psi$, magnetic vector potential $\boldsymbol{A}$, and magnetic field $\boldsymbol{B}$ according to the following transformations:

$$
\begin{aligned}
& x \longrightarrow l x, \\
& \psi \rightarrow \sqrt{\frac{|\alpha|}{\beta} \psi,} \\
& A \longrightarrow \sqrt{\frac{8 \pi \alpha^{2} l^{2}}{\beta}} A, \\
& B \longrightarrow \sqrt{\frac{8 \pi|\alpha|^{2}}{\beta}} B, \\
& \bar{\Phi} \longrightarrow \frac{|\alpha|}{2 \gamma e} \bar{\Phi}
\end{aligned}
$$

where $\gamma$ is relaxation parameter. For simplicity, we choose length scale in terms of coherence length (i.e. $l=\xi$ ). GinzburgLandau parameter $\kappa$ is defined as $\kappa=\lambda / \xi$. $\kappa$ defines the type of superconductor, $\kappa<1 / \sqrt{2}$ for type-I and $\kappa>1 / \sqrt{2}$ for type-II superconductors. Minimizer of Ginzburg-Landau energy functional (1) satisfies Euler-Lagrange equations, generally known as Ginzburg-Landau (GL) equations:

$$
\begin{aligned}
\frac{\partial \psi}{\partial t}+\iota \bar{\Phi} \psi= & \psi|\psi|^{2} \psi-\left(\iota \nabla+\frac{1}{\kappa} A\right)^{2} \psi \\
-\sigma\left(\frac{\partial A}{\partial t}+\kappa \nabla \bar{\Phi}\right)= & \nabla \times \nabla \times A+\frac{1}{\kappa^{2}}|\psi|^{2} A \\
& +\frac{\iota}{2 \kappa}\left(\psi^{*} \nabla \psi-\psi \nabla \psi^{*}\right) .
\end{aligned}
$$

$\sigma$ is electrical conductivity defined as $\sigma=\sigma_{n}\left(2 \pi \hbar / \gamma c^{2}\right)\left(l^{2} / \xi^{2}\right)$, while $\sigma_{n}$ is normal conductance. External magnetic field is assumed to be uniformly distributed over the sample surface. GL equations were solved using natural boundary conditions, i.e., supercurrent across the boundaries is zero and magnetic field at the boundaries is given as follows:

$$
\begin{aligned}
\left(\iota \nabla+\frac{1}{\kappa} \boldsymbol{A}\right) \psi \cdot \boldsymbol{n} & =0 \quad \text { on boundary } \Gamma \\
(\boldsymbol{\nabla} \times \boldsymbol{A}) \times \boldsymbol{n} & =\boldsymbol{B} \times \boldsymbol{n} \text { on boundary } \Gamma,
\end{aligned}
$$

where $\boldsymbol{n}$ is unit normal vector on the boundary $\Gamma$ of the region $\Omega$.

TDGL model is gauge invariant under transformation:

$$
\begin{aligned}
\psi^{\prime} & =\psi e^{\imath \kappa \chi} \\
A^{\prime} & =A+\nabla \chi \\
\bar{\Phi}^{\prime} & =\bar{\Phi}-\frac{\partial \chi}{\partial t}
\end{aligned}
$$


where gauge $\chi$ is a function of space and time. For the present study, we choose zero electric potential gauge $(\bar{\Phi}=0)$ known as Coulomb Gauge. So (3) and (4) reduce to

$$
\begin{aligned}
\frac{\partial \psi}{\partial t}= & \psi-|\psi|^{2} \psi-\left(\iota \nabla+\frac{1}{\kappa} A\right)^{2} \psi \\
-\sigma \frac{\partial A}{\partial t}= & \nabla \times \nabla \times A+\frac{1}{\kappa^{2}}|\psi|^{2} A \\
& +\frac{\iota}{2 \kappa}\left(\psi^{*} \nabla \psi-\psi \nabla \psi^{*}\right) .
\end{aligned}
$$

Energy density $H_{t o t}=H_{t o t}(x, y, z, t)$ in superconductor is distributed into three parts: superconducting energy density $H_{\text {sup }}=H_{\text {sup }}(x, y, z, t)$, interaction energy density $H_{\text {int }}=H_{\text {int }}(x, y, z, t)$, and magnetic energy density $H_{\text {mag }}=$ $H_{\text {mag }}(x, y, z, t)$ given as $[18,29]$

$$
\begin{aligned}
H_{\text {sup }} & =\frac{1}{\kappa^{2}}|\nabla \psi|^{2}-|\psi|^{2}+\frac{1}{2}|\psi|^{4} \\
H_{\text {int }} & =\frac{\iota}{\kappa} A\left((\nabla \psi) \psi^{*}-\psi\left(\nabla \psi^{*}\right)\right)+|A|^{2}|\psi|^{2} \\
H_{\text {mag }} & =(B-\nabla \times A)^{2},
\end{aligned}
$$

where length scales are taken in terms of London penetration depth (i.e. $l=\lambda)$. Total energy $\left(H_{t o t}\right)$ density is the sum of these three energy densities, $H_{\text {tot }}=H_{\text {sup }}+H_{\text {int }}+H_{\text {mag }}$; total energy is the integral of total energy density over the region $\Omega, G_{t o t}=$ $\int H_{\text {tot }}(x, y, z, t) d \Omega$.

Gauge invariant discretization [17, 30-33] is the most popular and widely used approach to solve TDGL equations, which is first-order accurate in time and secondorder accurate in space, and other finite element $[19,20]$, finite difference [34, 35], and spectral method [22] have also been developed. Link variable schemes $[16,17,36]$ are the most commonly used schemes to solve coupled GL equations. In the present work, in order to investigate carrier concentration, magnetization, and vortex dynamics in superconductor, we used coupled nonlinear TDGL equations (8) and (9) solved by finite difference scheme using link variables, described by Winiecki and Adams [16], implemented in three-dimensional code we have developed [18]. Equations (8) and (9) along with boundary condition equations (5) and (6) form the basis of the present work. The present work was performed close to critical temperature allowing the assumption of thermal suppression of surface barrier [3739]. The frequency of oscillating magnetic field was set to $0.00278 / t$ in nondimensionalized coordinates for the present work.

\section{Results and Discussion}

Three-dimensional, cubic superconductor domain of size $20 \xi \times 20 \xi \times 20 \xi$, periodic along $\mathrm{z}$-axis (along the direction of applied magnetic field), was discretized with grid size 0.5 $\xi$ in each direction, and magnetic field was applied in $\mathrm{z}$-axis direction with Ginzburg-Landau parameter $\kappa=4$. Initially, a constant magnetic field was applied, and on reaching

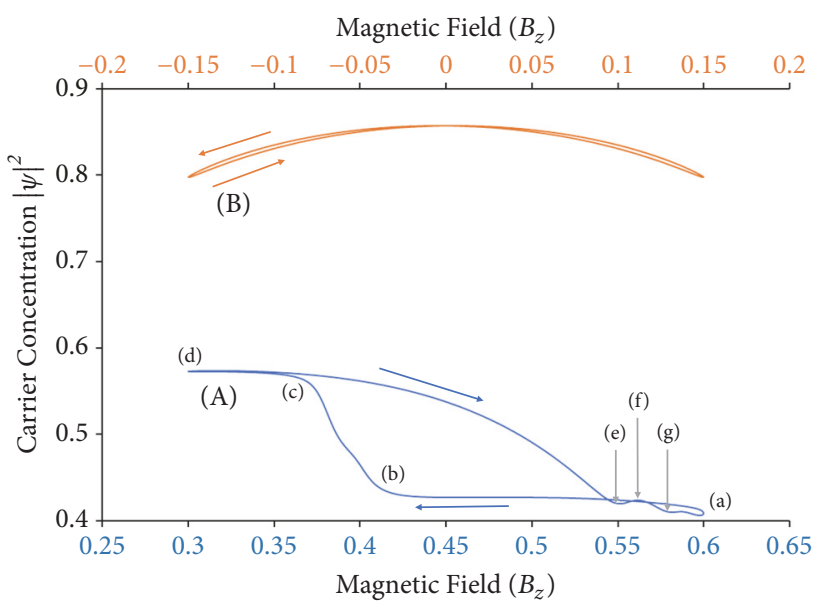

FIGURE 1: Variation of carrier concentration with magnetic field for cubic sample of side $20 \xi$ each, with parameters $\kappa=4$ and $\sigma=1$ under magnetic fields (A) $B_{z}=0.45 \kappa+0.15 \kappa \cos \theta$ and (B) $B_{z}=$ $0.15 \kappa \cos \theta$.

equilibrium state, it was allowed to oscillate as cosine wave. Cubic superconductor under two different types of magnetic field schemes, (A) $B_{z}=0.45 \kappa+0.15 \kappa \cos \theta$ and (B) $B_{z}=$ $0.15 \kappa \cos \theta$ (where $\theta=\omega t=2 \pi f t$ ), was simulated to investigate the effect of static background magnetic field on the superconductor in the presence of an oscillating magnetic field. The system was initialized with the completely superconducting sample $(|\psi|=1)$ and no magnetic vector potential $(A=0)$. The system was relaxed to minimize Gibbs free energy. During this energy minimization process, vortices entered the sample and relaxed their positions to minimum energy. Magnetic field oscillations were introduced as the sample reached minimum energy state, resulting in a new series of entrance and leaving of vortices, corresponding to different positions of magnetic field wave. Figure 1 shows a variation of average carrier concentration of the sample with magnetic field. The pattern of carrier concentration is symmetric for positive and negative half cycles but nonsymmetric for increasing and decreasing parts of magnetic field oscillation, shown in Figure 1 (for magnetic field of type (B)), indicating a lag in vortex dynamics behind the oscillating magnetic field. For the situation of magnetic field of type (A), the system is initially subjected to $B_{z}=0.6$, and after sufficient time, on reaching equilibrium state, a small oscillation with amplitude 0.15 in magnetic field was introduced.

During decaying half of magnetic field cycle (i.e., $\left|B_{z}\right|=$ $0.6 \longrightarrow 0.3$ ), carrier concentration does not increase as rapidly as decrease in magnetic field, till a certain value of magnetic field, where it is difficult for the sample to keep the vortices bound any further within the sample and a set of loosely bound vortices leave the sample, causing a sudden increase in carrier concentration shown by steps between positions (b) and (c) in Figure 1, carrier concentration peaks at minimum magnetic field, i.e., $B_{z}=0.3$. During increasing half of magnetic field cycle (i.e., $\left|B_{z}\right|=0.3 \longrightarrow 0.6$ ), penetration of magnetic field in large quantity starts from the sample edges till vortices are formed and they repel magnetic 


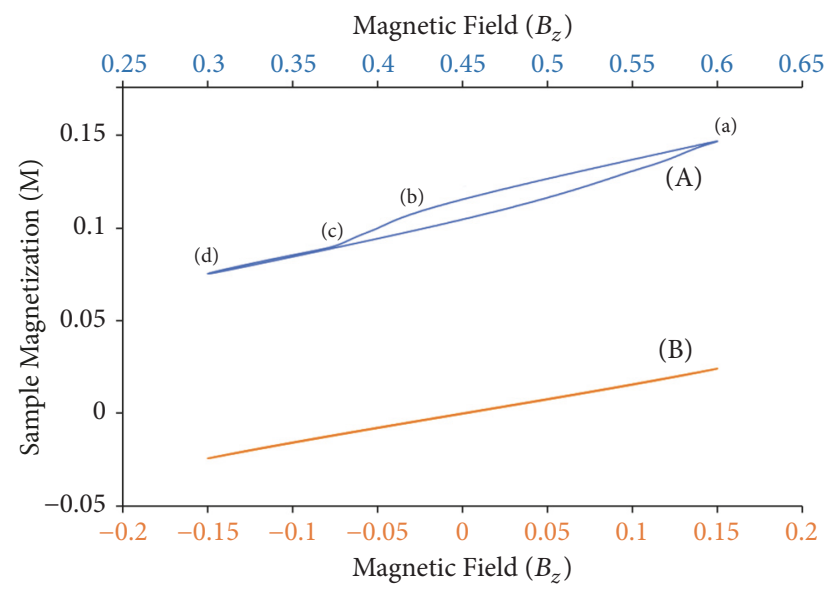

FIgURE 2: Magnetization $(M=\nabla \times A)$ vs. magnetic field $\left(B_{z}\right)$ plot for cubic sample of side $20 \xi$ each, with parameters $\kappa=4$ and $\sigma=1$ under magnetic field oscillations of the type $(A) B_{z}=0.45 \kappa+0.15 \kappa \cos \theta$ and (B) $B_{z}=0.15 \kappa \cos \theta$.

field penetrations from the edges. Relaxation of vortices causes an increase in carrier concentration indicated by sections (e) and (f) in Figure 1, and formation and relaxation of the first additional set of vortices are indicated by this small peak; the same process repeats itself for every subsequent set of vortices entering the sample indicated by $(\mathrm{g})$ in Figure 1.

Energy dissipation $\left(E_{d}\right)$ in magnetic oscillations is the area covered by hysteresis loop ( $M-H$ curve) of sample magnetization with magnetic field, which takes the following form:

$$
E_{d}=\Omega \oint \boldsymbol{B}_{z} \cdot d \boldsymbol{M}
$$

where $M$ is sample magnetization $(\boldsymbol{M}=\boldsymbol{\nabla} \times \boldsymbol{A}), \Omega$ is volume bounded by the superconductor, and $B_{z}$ is the applied external magnetic field. This type of behavior of magnetic field trapped inside the superconductor lagging behind the magnetic field oscillations indicates the energy dissipation during the cycle. The area of loop, attributed to the energy dissipated during a cycle, was observed to increase with frequency of magnetic oscillations, due to limited velocities of vortices. Figure 2 shows variation of average sample magnetization $(\boldsymbol{\nabla} \times \boldsymbol{A})$ with external magnetic field oscillations at normalized frequency $f=0.00278 / t$. It was observed that during decreasing and increasing half cycles of magnetic field oscillations, sample magnetization behaved in a similar way (comparing with plots behavior) to that of carrier concentration in Figure 1. During decreasing part of magnetic field sample magnetization had a rapid drop between (b) and (c) in Figure 2 at the same magnetic field intensity as observed between (b) and (c) in Figure 1. This behavior of a sudden drop in magnetization, between (b) and (c) in Figure 2, and sudden rise in carrier concentration, between (b) and (c) in Figure 1, represent the same process and are attributed to the process of a set of vortices leaving the sample. This trapped energy can be anticipated to transform in other forms of energy, mostly heat, which accumulates with a number of cycles, resulting in thermal quenching of the superconductor.

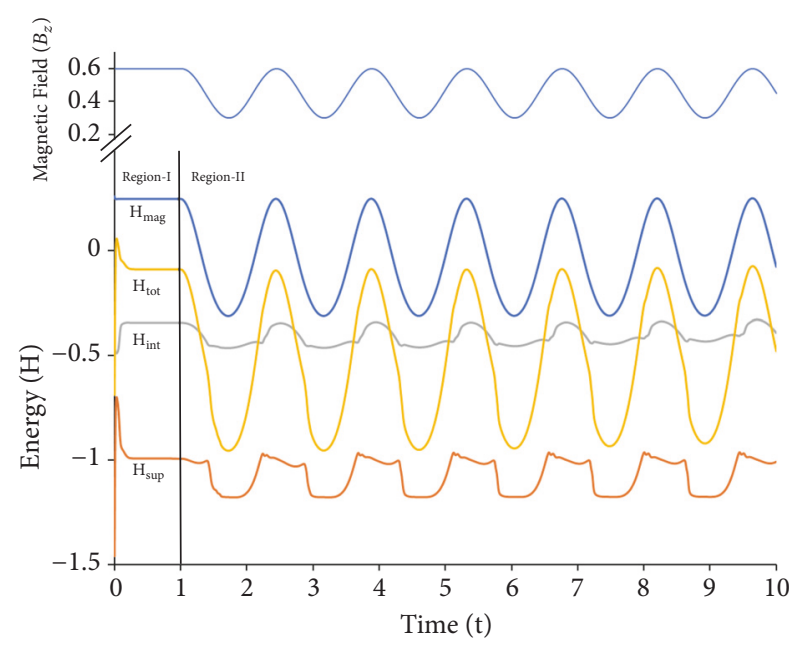

Figure 3: Temporal distribution of energy densities of cubic superconducting sample in static and oscillating magnetic fields, for cubic sample of side $20 \xi$ each, with parameters $\kappa=4$ and $\sigma=1$, and $B_{z}=0.45 \kappa+0.15 \kappa \cos \theta$.

It can also be inferred that, at faster vehicle speeds, resulting in higher frequencies, this energy accumulation may result in a rapid quenching of superconductor. This energy loss during a cycle was observed to be $\sim 10$ times greater in the presence of additional static background magnetic field (type A) compared with that in its absence (type B), which can be seen by the area swept by both curves in Figure 2 .

Temporal distribution of three energy components and total energy for the sample under the magnetic field of type (A) is shown in Figure 3. Initially, (at $t=0$ ) sample was in a nonequilibrium state with no magnetic field penetration and carrier concentration at maximum. Nonequilibrium state of magnetic field distribution across the boundary is the reason of large initial value of $H_{\text {mag }}$, but as the field penetration starts, $H_{\text {mag }}$ rapidly drops to equilibrium values, because the number of vortices will remain the same before introduction of field oscillations and there would only be rearrangement of vortices in the sample, and $H_{\text {mag }}$ do not change during the rearrangement of vortices. Initially, due to uniform penetration of vortices from the boundaries, $H_{\text {sup }}$ increases rapidly because average carrier concentration decreases and the second term in (10) dominates. As the vortices are formed and start relaxing, $H_{\text {sup }}$ starts decreasing forming an initial hump-like structure, indicating nonvortex to vortex transition. $H_{\text {int }}$ is responsible for the interaction of magnetic and superconducting energies. The second term in (11) is responsible for the formation of vortices while the first term controls vortex dynamics inside the superconductor.

Figure 3 (region II) represents all the three component energy densities and total energy density with the magnetic field of type (A). As system reaches equilibrium, oscillations in magnetic field are introduced as cosine wave, in the range $0.6 \kappa \geq B_{z} \geq 0.3 \kappa$. In region II as $B_{z}$ decreases, the balance between external magnetic field and vortex magnetization is disturbed, resulting in an increase in intervortex spacing and exit from the sample, and all the energies start oscillating with 


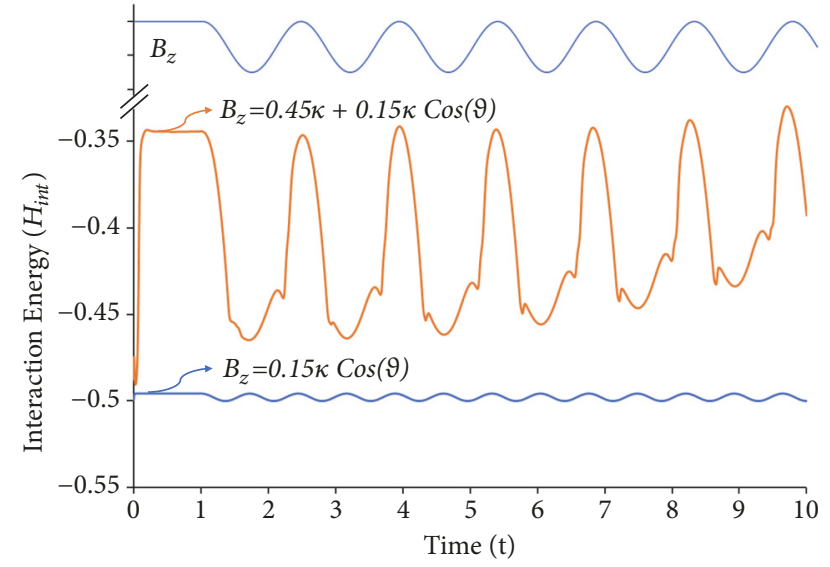

FIgURE 4: Temporal distribution of interaction energy densities of cubic superconducting sample in the magnetic field of types (A) $B_{z}=0.45 \kappa+0.15 \kappa \cos \theta$ and (B) $B_{z}=0.15 \kappa \cos \theta$, for cubic sample of side $20 \xi$ each, with parameters $\kappa=4$ and $\sigma=1$.

the oscillations in $B_{z}$, and the process of repeated entrance and exit of vortices starts. Peak shape analysis of $H_{\text {sup }}$ and $H_{\text {int }}$ shows small kinks in energies, indicating the moments when outermost (or least bound) vortices leave the sample; after that, a sudden dip was observed in $H_{\text {sup }}$ and $H_{\text {int }}$ because the remaining vortices which are now free from force exerted by the vortices just left and relaxed themselves; this kink is also visible in total energy pattern, similar to the one observed at positions (b) and (c) in Figures 1 and 2. Interestingly, energy patterns of $H_{\text {sup }}$ and $H_{\text {int }}$ are not symmetric for increasing and decreasing half cycles of $B_{z}$, i.e., entrance and exit of vortices. Peak shape analysis shows a quick entrance and slow exit of vortices during increasing and decreasing half cycles of $B_{z}$, resulting in hysteresis-type behavior and energy accumulation which have already been discussed. An irregularity in $H_{\text {int }}$ is observed after a number of cycles, due to energy accumulation. This effect was only observed in $H_{\text {int }}$ due to its dependence on vortex dynamics, having limited velocities, resulting in $H_{\text {int }}$ reaching equilibrium value with a delay compared to $H_{\text {mag }}$ and $H_{\text {sup }}$. Repeated entrance and leaving of vortices over a cycle do not give sufficient time to the sample to completely attain equilibrium state, resulting in accumulation of energy in the sample in the form of interaction energy. This accumulated energy represented the area enclosed by magnetic field and sample magnetization shown in Figure 2, which may appear in the form of heat resulting in thermal quenching of superconductor in practical applications. This effect is significantly high ( 10 times) for the samples under magnetic field of type (A) compared to the samples under magnetic field of type (B). Figure 4 shows the comparison of $H_{\text {int }}$ of the systems with and without steady magnetic field (of types (A) and (B)) under the same oscillating component of magnetic field. It was observed (by the comparison of interaction energies in Figure 4) that, in the presence of steady background magnetic field, the energy dissipation (or the abnormalities in $H_{\text {int }}$ ) is $\sim 10$ times higher than that observed for the case without additional external magnetic field. Therefore, a static background magnetic field plays a significant role in addition to oscillating magnetic field in thermal dissipation and subsequently quenching of superconductor moving on PM guideway in a Maglev vehicle system; the rate of such energy dissipation/accumulation is directly dependent on the speed of the vehicle.

\section{Conclusions}

Time-dependent Ginzburg-Landau model was used to investigate type-II superconductor under the oscillating magnetic field, with additional static background magnetic field. Finite difference scheme was used for cubic superconducting sample. Carrier concentration, magnetization, and energy in the presence and absence of static background magnetic field were studied. Nonuniform behavior in carrier concentration with the oscillating magnetic field was observed and was related to vortex dynamics on point-to-point basis. Lagging of average sample magnetization behind magnetic field was observed during magnetic field oscillations. Area of hysteresis loop was measured and was attributed to dissipated energy in the sample, accumulated over time. The same effect caused anomalies in interaction energy density of the sample under magnetic field oscillations. Ripples in superconducting energy density were observed due to entrance and exit of vortices during magnetic field oscillations, which is the result of the same effect of small peaks (and sudden rise) observed in average carrier concentration with increasing (and decreasing) external magnetic field magnitude. Comparison between energy patterns in the presence and absence of background steady magnetic field indicated that more energy is dissipated in the presence of background magnetic field than that in its absence, suggesting that, in practical devices (e.g., Maglev vehicles) when magnetic field is oscillating (magnetic guideways with defects), its necessary to shield the superconductor from background magnetic fields to avoid energy dissipation and thermal quenching of the superconductor.

\section{Data Availability}

All the results published in this article can be reproduced by using equations and parameters described herein.

\section{Conflicts of Interest}

The authors declare that there are no conflicts of interest regarding the publication of this paper.

\section{Acknowledgments}

This work was supported by National Science Foundation of China, Grant nos. 11174030 and 11504020 . Author Hasnain Mehdi Jafri acknowledges the Higher Education Commission of Pakistan for the Ph.D. scholarship.

\section{Supplementary Materials}

Figure S1: vortex structure of the sample at (a) $\mathrm{Bz}=0.6 \kappa$ (oscillating magnetic field), (b) $\mathrm{Bz}=0.3 \kappa$ (oscillating magnetic 
field), (c) $\mathrm{Bz}=0.15 \kappa$ (oscillating magnetic field), and (d) $\mathrm{Bz}=0.15 \kappa$ (nonoscillating magnetic field) for cubic sample of size $20 \xi \times 20 \xi \times 20 \xi$, with parameters $\kappa=4$ and $\sigma=1$. (Supplementary Materials)

\section{References}

[1] J. Bardeen, L. N. Cooper, and J. R. Schrieffer, "Microscopic theory of superconductivity," Physical Review A: Atomic, Molecular and Optical Physics, vol. 106, article 162, 1957.

[2] P. W. Anderson, "Plasmons, gauge invariance, and mass," Physical Review A: Atomic, Molecular and Optical Physics, vol. 130, no. 1, pp. 439-442, 1963.

[3] F. Englert and R. Brout, "Broken symmetry and the mass of gauge vector mesons," Physical Review Letters, vol. 13, no. 9, article 321, 1964.

[4] G. S. Guralnik, C. R. Hagen, and T. W. B. Kibble, "Global conservation laws and massless particles," Physical Review Letters, vol. 13, no. 20, pp. 585-587, 1964.

[5] P. W. Higgs, "Broken symmetries and the masses of gauge bosons," Physical Review Letters, vol. 13, pp. 508-509, 1964.

[6] W. Meissner and R. Ochsenfeld, "Ein neuer Effekt bei Eintritt der Supraleitfähigkeit," Naturwissenschaften, vol. 21, no. 44, pp. 787-788, 1933.

[7] M. C. N. Fiolhais, H. Esśen, C. Providencia, and A. B. Nordmark, "Magnetic field and current are zero inside ideal conductors," Progress in Electromagnetics Research B, no. 27, pp. 187-212, 2011.

[8] H. Essén and M. C. N. Fiolhais, "Meissner effect, diamagnetism, and classical physics-a review," American Journal of Physics, vol. 80, no. 2, pp. 164-169, 2012.

[9] A. A. Abrikosov, "Magnetic properties of superconductors of the second group," Journal of Experimental and Theoretical Physics, vol. 5, no. 6, 1957.

[10] P. L. Gammel, D. J. Bishop, G. J. Dolan et al., "Observation of hexagonally correlated flux quanta in YBa2Cu3O7," Physical Review Letters, vol. 59, no. 22, pp. 2592-2595, 1987.

[11] H. F. Hess, R. B. Robinson, R. C. Dynes, J. M. Valles Jr., and J. V. Waszczak, "Scanning-Tunneling-Microscope Observation of the Abrikosov Flux Lattice and the Density of States near and inside a Fluxoid," Physical Review Letters, vol. 62, no. 2, pp. 214216, 1989.

[12] U. Essmann and H. Träuble, "The direct observation of individual flux lines in type II superconductors," Physics Letters A, vol. 24, no. 10, pp. 526-527, 1967.

[13] M. Karmakar, "Electrostatic potential in high-temperature superconducting cuprates: Extended ginzburg-landau theory," Advances in Condensed Matter Physics, vol. 2011, 2011.

[14] T. Kidanemariam and G. Kahsay, "Theoretical Study of Upper Critical Magnetic Field (HC2) in Multiband Iron Based Superconductors," Advances in Condensed Matter Physics, vol. 2016, Article ID 5470429, 10 pages, 2016.

[15] L. P. Gor'kov, "Microscopic derivation of the Ginzburg-Landau equations in the theory of superconductivity," Journal of Experimental and Theoretical Physics, vol. 9, no. 6, pp. 1364-1367, 1959.

[16] T. Winiecki and C. S. Adams, "A fast semi-implicit finitedifference method for the TDGL equations," Journal of Computational Physics, vol. 179, no. 1, pp. 127-139, 2002.

[17] W. D. Gropp, H. G. Kaper, G. K. Leaf, D. M. Levine, M. Palumbo, and V. M. Vinokur, "Numerical simulation of vortex dynamics in type-II superconductors," Journal of Computational Physics, vol. 123, no. 2, pp. 254-266, 1996.

[18] H. M. Jafri, X. Ma, C. Zhao et al., "Numerical simulation of vortex dynamics in type-II superconductors in oscillating magnetic field using time-dependent Ginzburg-Landau equations," Journal of Physics: Condensed Matter, vol. 29, no. 50, p. 505701, 2017.

[19] Q. Du, "Finite element methods for the time-dependent Ginzburg-Landau model of superconductivity," Computers \& Mathematics with Applications, vol. 27, no. 12, pp. 119-133, 1994.

[20] Q. Du, M. D. Gunzburger, and J. S. Peterson, "Solving the Ginzburg-Landau equations by finite-element methods," Physical Review B: Condensed Matter and Materials Physics, vol. 46, no. 14, pp. 9027-9034, 1992.

[21] B. Li and Z. Zhang, "A new approach for numerical simulation of the time-dependent Ginzburg-Landau equations," Journal of Computational Physics, vol. 303, pp. 238-250, 2015.

[22] L. Q. Chen and J. Shen, "Applications of semi-implicit Fourierspectral method to phase field equations," Computer Physics Communications, vol. 108, no. 2-3, pp. 147-158, 1998.

[23] M. Liu, S. Wang, J. Wang, and G. Ma, "Effect of AC magnetic field on the levitation force of YBCO bulk above NdFeB guideway," Journal of Low Temperature Physics, vol. 155, no. 34, pp. 169-176, 2009.

[24] J. Wang, S. Wang, Y. Zeng et al., "The first man-loading high temperature superconducting Maglev test vehicle in the world," Physica C: Superconductivity and its Applications, vol. 378-381, pp. 809-814, 2002.

[25] L. Zhang, J. Wang, S. Wang, and Q. He, "Influence of AC external magnetic field perturbation on the guidance force of HTS bulk over a NdFeB guideway," Physica C: Superconductivity and its Applications, vol. 459, no. 1-2, pp. 43-46, 2007.

[26] J. Barba-Ortega and E. Sardella, "Superconducting properties of a mesoscopic parallelepiped with anisotropic surface conditions," Physics Letters A, vol. 379, no. 47-48, pp. 3130-3135, 2015.

[27] J. Barba-Ortega, E. Sardella, and J. A. Aguiar, "Superconducting properties of a parallelepiped mesoscopic superconductor: A comparative study between the 2D and 3D Ginzburg-Landau models," Physics Letters A, vol. 379, no. 7, pp. 732-737, 2015.

[28] L. P. GoR'Kov and G. M. Eliashberg, "Generalization of the Ginzburg-Landau equations for non-stationary problems in the case of alloys with paramagnetic impurities," Soviet Journal of Experimental and Theoretical Physics, vol. 27, p. 328, 1968.

[29] T. S. Alstrøm, M. P. Sørensen, N. F. Pedersen, and S. r. Madsen, "Magnetic flux lines in complex geometry type-II superconductors studied by the time dependent Ginzburg-Landau equation," Acta Applicandae Mathematicae, vol. 115, no. 1, pp. 63-74, 2011.

[30] H. Frahm, S. Ullah, and A. T. Dorsey, "Flux dynamics and the growth of the superconducting phase," Physical Review Letters, vol. 66, no. 23, pp. 3067-3070, 1991.

[31] R. Kato, Y. Enomoto, and S. Maekawa, "Computer simulations of dynamics of flux lines in type-II superconductors," Physical Review B: Condensed Matter and Materials Physics, vol. 44, no. 13, pp. 6916-6920, 1991.

[32] M. MacHida and H. Kaburaki, "Direct simulation of the timedependent Ginzburg-Landau equation for type-II superconducting thin film: Vortex dynamics and V-I characteristics," Physical Review Letters, vol. 71, no. 19, pp. 3206-3209, 1993.

[33] G. W. Crabtree, D. O. Gunter, H. G. Kaper, A. E. Koshelev, G. K. Leaf, and V. M. Vinokur, "Numerical simulations of driven vortex systems," Physical Review B: Condensed Matter and Materials Physics, vol. 61, no. 2, pp. 1446-1455, 2000. 
[34] E. Coskun and M. K. Kwong, "Simulating vortex motion in superconducting films with the time-dependent GinzburgLandau equations," Nonlinearity, vol. 10, no. 3, pp. 579-593, 1997.

[35] J. F. Blackburn, A. Campbell, and E. K. H. Salje, "The force-free case in three-dimensional superconductors: A computational study," Philosophical Magazine, vol. 80, no. 8, pp. 1455-1471, 2000.

[36] J. Barba-Ortega, E. Sardella, and J. A. Aguiar, "Superconducting boundary conditions for mesoscopic circular samples," Superconductor Science and Technology, vol. 24, no. 1, 2011.

[37] R. Córdoba, T. I. Baturina, J. Sesé et al., "Magnetic fieldinduced dissipation-free state in superconducting nanostructures," Nature Communications, vol. 4, no. 1, p. 1437, 2013.

[38] C. P. Bean and J. D. Livingston, "Surface barrier in type-II superconductors," Physical Review Letters, vol. 12, no. 1, pp. 1416, 1964.

[39] W. V. Pogosov, "Thermal suppression of surface barrier in ultrasmall superconducting structures," Physical Review B: Condensed Matter and Materials Physics, vol. 81, no. 18, 2010. 

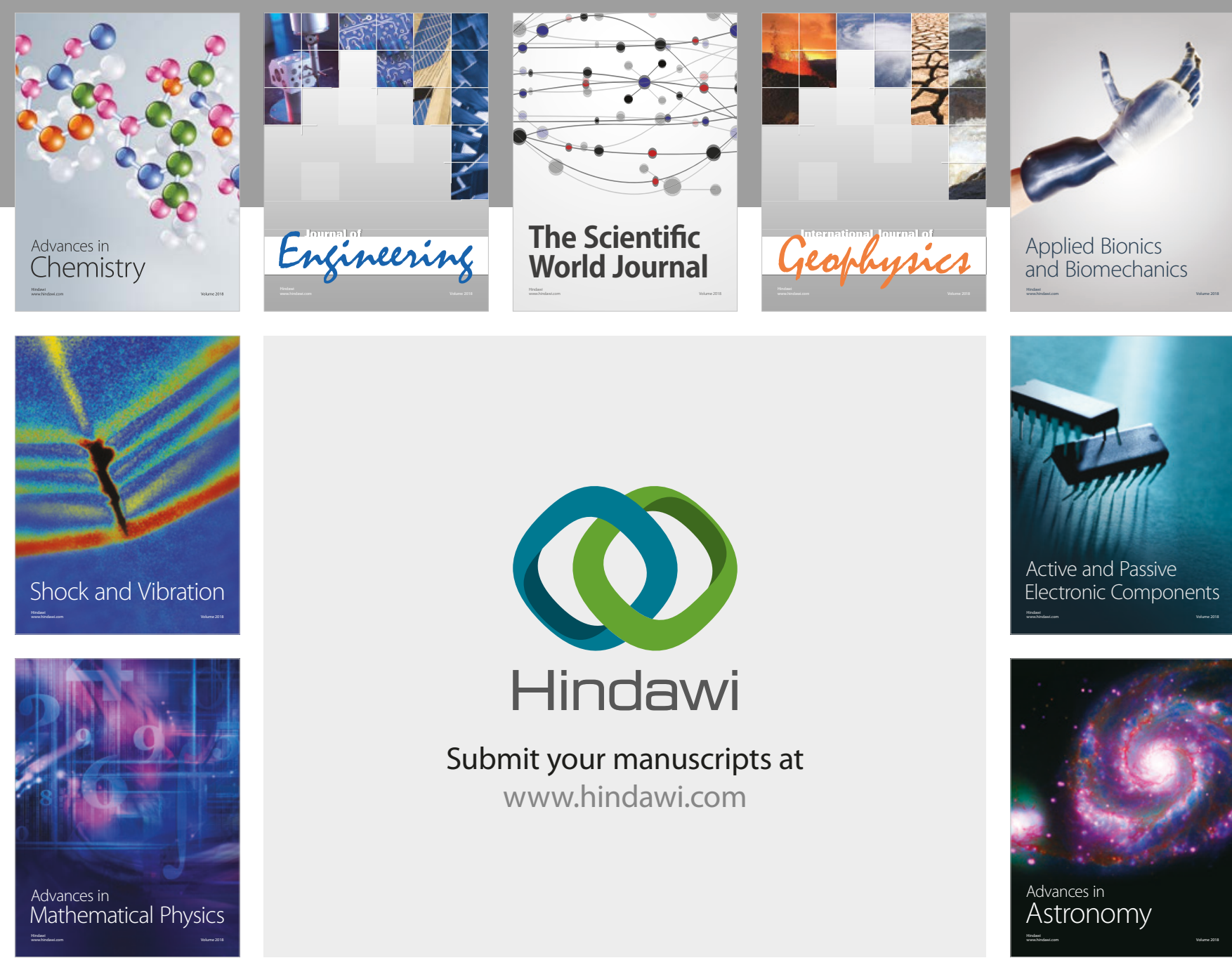

Submit your manuscripts at

www.hindawi.com

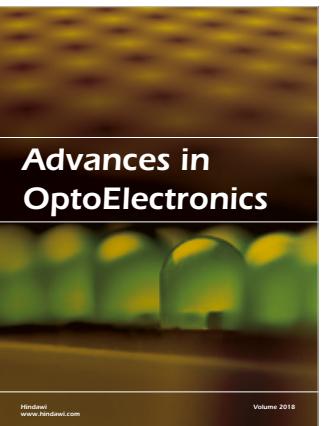

\section{Rotcting Machinery}
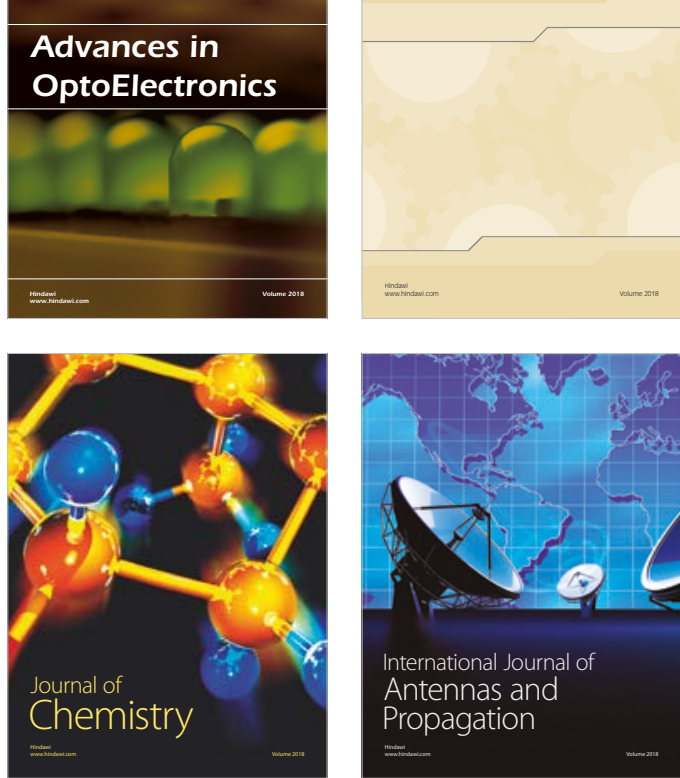

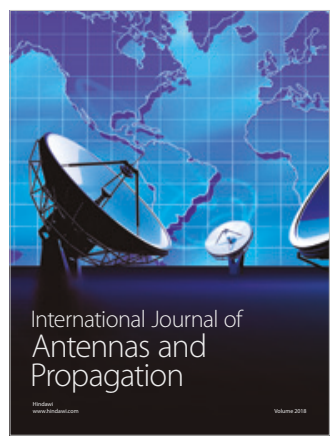

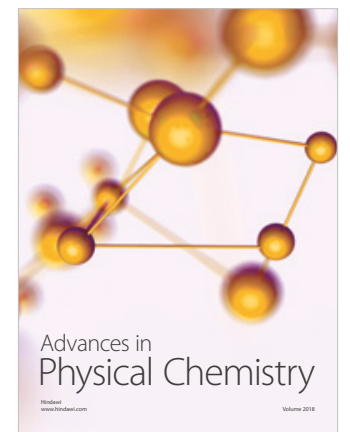

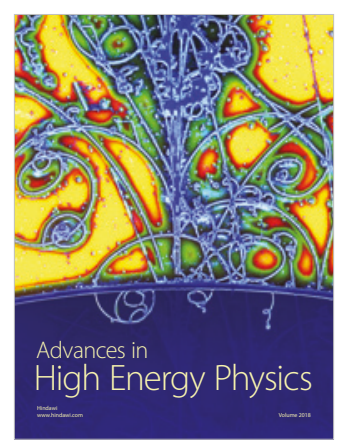

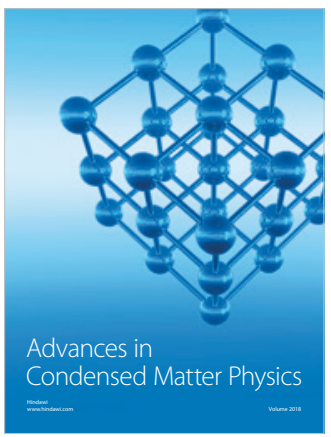

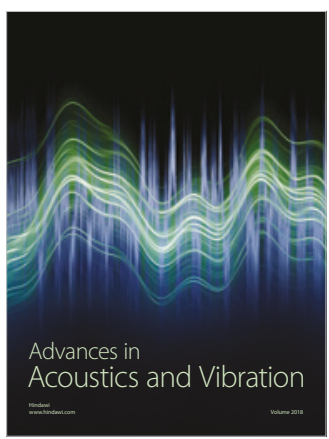

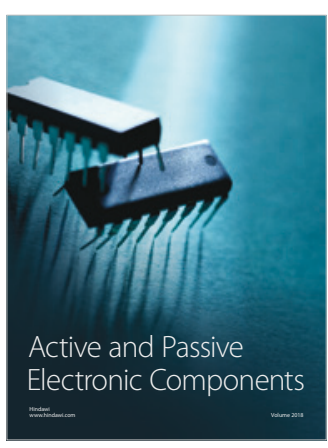
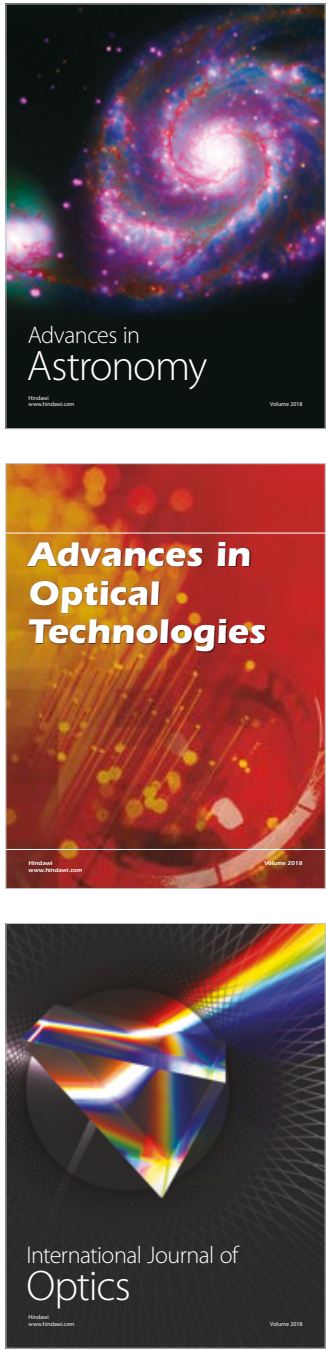